\title{
Pacific
}

Journal of

Mathematics

\section{THE FORMAL GROUP OF THE JACOBIAN OF AN} ALGEBRAIC CURVE

\author{
MARGaRet N. FREIJE
}




\title{
THE FORMAL GROUP OF THE JACOBIAN OF AN ALGEBRAIC CURVE
}

\author{
MARgaret N. Freije
}

\begin{abstract}
In this paper we give an explicit construction of the formal group of the Jacobian of an algebraic curve using a basis for the holomorphic differentials on the curve at a rational non-Weierstrass point. We construct the formal group of the Jacobian of the modular curve $X_{0}(l)$ and using a result of $T$. Honda, we prove that this formal group is $p$ integral for all but finitely many $p$.
\end{abstract}

Introduction. Formal group laws have proven to be very useful tools in many areas of mathematics and computer science. In particular, the formal group of an elliptic curve has been used to great effect in elliptic curve theory (for details see for example Silverman [13]) and the use of the formal group of an abelian variety is pervasive in arithmetic and algebraic geometry (see Shatz [11] or Milne [8]). Despite the fact that explicit formulae have been useful in the elliptic curve case, explicit examples of the formal group of other abelian varieties are few.

Recently, Grant [4] and Flynn [3] have independently given explicit constructions of the formal group of the Jacobian of curves of genus two. Grant uses classical formulae for genus two theta functions to give explicit defining equations for the Jacobian and a set of parameters for its group law in a specific $\mathbb{P}^{8}$ embedding. His embedding requires that the curve have a Weierstrass point defined over the base field. Flynn's result does not assume the existence of a rational Weierstrass point and thus he must use a $\mathbb{P}^{15}$ embedding of the Jacobian. This paper gives an explicit construction of the formal group of the Jacobian using a basis for the holomorphic differentials on the curve. The construction generalizes that of the formal group of an elliptic curve.

In $\S$ I, we review some of the basic facts about higher dimensional formal groups. Hazewinkel [5] is a good reference for the theory of formal groups in general. Section II details the construction of the formal group of the Jacobian. Since this construction depends only on a basis for the holomorphic differentials on the curve at a nonWeierstrass point, it is especially useful in cases where much is known 
about these differentials. In $\S I I I$, we give an example of just such a case and construct the formal group of the Jacobian of the modular curve $X_{0}(l), l$ a prime. Using a result of $\mathrm{T}$. Honda, and the connection between the differentials on this curve and the Fourier expansions of cusp forms of weight 2 on $\Gamma_{0}(l)$ we prove that this formal group is $p$-integral for all but finitely' many primes $p$.

The author wishes to thank the referee for many helpful suggestions on the styles and organization of this paper.

I. Higher dimensional formal groups. Let $R$ be a commutative ring with identity and let $R[[X]], X=\left(x_{1}, \ldots, x_{n}\right)$, be the power series ring in $n$ variables over $R$. If $f(X)$ and $g(X) \in R[[X]]$ and $n$ is a positive integer, we say $f(X) \equiv g(X)(\bmod \operatorname{deg} n)$ if $f(X)-g(X)$ has no terms of total degree less than $n$.

Definition 1. A formal group law $F(X, Y)$ of dimension $n$ over $R$ is an $n$-tuple of power series $\left(F_{1}(X, Y), \ldots, F_{n}(X, Y)\right), F_{i}(X, Y)$ $\in R[[X, Y]]$, satisfying:

(i) $F(X, 0)=X, F(0, Y)=Y$,

(ii) $F(X, F(Y, Z))=F(F(X, Y), Z)$.

It is an easy exercise to show that one has formal inverses, that is there is an $n$-tuple of power series $l(X)=\left(l_{1}(X), \ldots, l_{n}(X)\right), l_{j}(X) \in$ $R[[X]]$, satisfying $l(X) \equiv-X(\bmod \operatorname{deg} 2)$ and $F(X, l(X))=0$.

If $F(X, Y)=F(Y, X)$ we say $F$ is a commutative formal group.

Definition 2. (a) If $F(X, Y)$ is a formal group of dimension $n$ and $G(X, Y)$ is a formal group of dimension $m$ then $\alpha: F \rightarrow G$ is a formal group homomorphism over $R$ if $\alpha(X)=\left(\alpha_{1}(X), \ldots, \alpha_{m}(X)\right)$ is an $m$-tuple of power series, $\alpha_{i}(X) \in X R[[X]]$, satisfying $\alpha(F(X, Y))=G(\alpha(X), \alpha(Y))$.

(b) If $\alpha: F \rightarrow G$ is a formal group homomorphism over $R$, then $\alpha$ is a formal group isomorphism over $R$ if and only if there is a formal group homomorphism $\beta: G \rightarrow F$ over $R$ such that $\alpha(\beta(Y))=Y$ and $\boldsymbol{\beta}(\boldsymbol{\alpha}(X))=X$.

It can be shown that $\alpha: F \rightarrow G$ is a formal group isomorphism over $R$ if and only if $\operatorname{dim} F=\operatorname{dim} G$ and the Jacobian matrix of $\alpha$, $J(\boldsymbol{\alpha})=\left(\left(\partial \boldsymbol{\alpha}_{i} / \partial x_{j}\right)(0)\right)$ is invertible over $R$.

EXAMPLES. 1. The additive group of dimension $n, \mathbb{G}_{a}(X, Y)$ :

$$
\mathbb{G}_{a}(X, Y)=\left(x_{1}+y_{1}, \ldots, x_{n}+y_{n}\right) \text {. }
$$

2. The multiplicative group of dimension $n, \mathbb{G}_{m}(X, Y)$ :

$$
\mathbb{G}_{m}(X, Y)=\left(x_{1}+y_{1}+x_{1} y_{1}, \ldots, x_{n}+y_{n}+x_{n} y_{n}\right) \text {. }
$$


3. Let $\alpha(X)=\left(\ln \left(1+x_{1}\right), \ln \left(1+x_{2}\right), \ldots, \ln \left(1+x_{n}\right)\right)$. Then $\alpha$ is a formal group isomorphism over $\mathbb{Q}$ between $\mathbb{G}_{m}$ and $\mathbb{G}_{a}$, i.e.,

$$
\begin{aligned}
\alpha\left(\mathbb{G}_{m}(X, Y)\right) & =\mathbb{G}_{a}(\alpha(X), \alpha(Y))=\alpha(X)+\alpha(Y) \text { and } \\
J(\alpha) & =n \times n \text { identity matrix. }
\end{aligned}
$$

The isomorphism $\alpha$ is an example of a more general fact. If $R$ is a $\mathbb{Q}$-algebra and $F(X, Y)$ is a commutative formal group over $R$ of dimension $n$ then there is a formal group isomorphism between $F$ and the additive group of dimension $n$. We now show briefly how to construct this isomorphism.

Let $\Omega=\sum R[[X]] d x_{1}$ be the space of differential 1-forms where $d$ is the total derivative map from $R[[X]]$ to $\Omega$. If $\omega=\sum_{i=1}^{n} \varphi_{i}(X) d x_{i}$, $\varphi_{i}(X) \in R[[X]]$ is in $\Omega$, then you can get a new differential by evaluating $\omega(a)=\sum_{i=1}^{n} \varphi_{i}(a) d x_{i}$ for any $a \in R^{n}$.

We define $\omega=\sum_{i=1}^{n} \varphi_{i}(X) d x_{i}, \varphi_{i}(X) \in R[[X]]$, to be translation invariant if $\omega(F(X, T))=\sum_{i=1}^{n} \varphi_{i}(F(X, T)) d F(X, T)=\omega$. Thus we have $\omega$ is invariant if and only if

$$
\begin{aligned}
& \left(\varphi_{1}(F(X, T)), \ldots, \varphi_{n}(F(X, T))\right)\left(\frac{\partial F_{i}}{\partial x_{j}}(X, T)\right) \\
& \quad=\left(\varphi_{1}(X), \ldots, \varphi_{n}(X)\right) .
\end{aligned}
$$

Evaluating at $X=0$ clearly implies

$$
\left(\varphi_{1}(T), \ldots, \varphi_{n}(T)\right)=\left(\varphi_{1}(0), \ldots, \varphi_{n}(0)\right)\left(\frac{\partial F_{i}}{\partial x_{j}}(0, T)\right)^{-1}
$$

On the other hand if we use the associative law for $F$, differentiate $F_{i}(Y, F(X, T))=F_{i}((F(Y, X), T))$ with respect to $y_{j}$ and then set $Y=0$, we have:

$$
\left(\frac{\partial F_{i}}{\partial x_{j}}(0, F(X, T))\right)=\left(\frac{\partial F_{i}}{\partial x_{j}}(X, T)\right)\left(\frac{\partial F_{i}}{\partial x_{j}}(0, X)\right) .
$$

Substituting $F(X, T)$ for $T$ in (2) and using (3) we have (2) is equivalent to (1).

We have therefore that $\omega$ is invariant if and only if

$$
\begin{gathered}
\omega=\left(a_{1}, \ldots, a_{n}\right)\left(\frac{\partial F_{i}}{\partial x_{j}}(0, X)\right)^{-1}\left(\begin{array}{c}
d x_{1} \\
\vdots \\
d x_{n}
\end{array}\right) \\
\text { where } \omega(0)=a_{1} d x_{1}+\cdots+a_{n} d x_{n} .
\end{gathered}
$$


Thus the space of invariant differentials of $F$ is an $R$ module of rank $n$.

There is also a map $d$ from $\Omega$ to the space of differential 2-forms $\Omega^{2}$. If $R$ is a $\mathbb{Q}$-algebra, $d \omega=0$ implies $\omega=d f$ for some $f \in$ $R[[X]]$. If $F$ is a commutative formal group over $R$ and $\omega$ is an invariant differential of $F$ then $d \omega=0$ (Proposition 1.3, Honda [6]) and thus if $R$ is a $\mathbb{Q}$-algebra $\omega=d f, f \in R[[X]]$.

Let $F$ be a commutative formal group defined over a $\mathbb{Q}$-algebra $R$ and let $\omega_{1}, \ldots, \omega_{n}$ be a basis for the invariant differentials of $F$ over $R$. Let $\mathscr{L}_{i}(X) \in R[[X]]$ be the unique power series such that

$$
d \mathscr{L}_{i}=\sum_{j=1}^{n} \frac{\partial \mathscr{L}_{i}}{\partial x_{j}}(X) d x_{j}=\omega_{i} \quad \text { and } \quad \mathscr{L}_{i}(0)=0
$$

We then have the following well-known theorem:

THEOREM 1. Let $F$ be a commutative formal group of dimension $n$ defined over a $\mathbb{Q}$-algebra $R$. Let $\mathscr{L}(X)=\left(\mathscr{L}_{1}(X), \ldots, \mathscr{L}_{n}(X)\right)$ with $\mathscr{L}_{i}(X)$ defined as above. Then $\mathscr{L}(X)$ is a formal group isomorphism $\mathscr{L}: F \rightarrow \mathbb{G}_{a}^{n}$ from $F$ to the additive group of dimension $n$.

Proof. $\omega_{i}$ is an invariant differential of $F$; thus $\omega_{i}(F(X, T))=$ $\omega_{i}(X)$ over $R[[T]]$. This implies that $\mathscr{L}_{i}(F(X, T))=\mathscr{L}_{i}(X)+$ $c(T)$ where $c(T) \in R[[T]]$. Evaluating at $X=0$ we get $c(T)=$ $\mathscr{L}_{i}(F(0, T))=\mathscr{L}_{i}(T)$. Thus $\mathscr{L}$ is a formal group homomorphism from $F$ to $\mathbb{G}_{a}^{n}$ over $R$. The Jacobian matrix of $\mathscr{L}, J(\mathscr{L})=\left(a_{i j}\right)$ where $\omega_{i}(0)=\sum a_{i j} d x_{j}$. This is invertible over $F$ since $\omega_{1}, \ldots, \omega_{n}$ is an $R$-basis. Thus $\mathscr{L}$ is a formal group isomorphism over $R$ and $F(X, Y)=\mathscr{L}^{-1}(\mathscr{L}(X)+\mathscr{L}(Y))$.

The isomorphism $\mathscr{L}$ is called a logarithm of $F$. If $J(\mathscr{L})=$ the identity matrix, then $\mathscr{L}$ is called the strict logarithm of $F$.

\section{The formal group of the Jacobian of an algebraic curve.}

Notation. If $I=\left(i_{1}, \ldots, i_{g}\right)$ is an index set of nonnegative integers, let $I$ ! denote $i_{1} ! i_{2} ! \cdots i_{g} !, N_{I}=i_{1}+2 i_{2}+\cdots+g i_{g}$ and

$$
B(I)=\frac{(-1)^{i_{2}+i_{4}+\cdots}\left(i_{1}+i_{2}+\cdots+i_{g}-1\right) !}{I !} .
$$


For any $k=1, \ldots, g, i_{k} B(I)$ is a multinomial coefficient and thus is an integer. If $X=\left(x_{1}, \ldots, x_{g}\right)$ is a $g$-tuple of variables, let $X^{I}$ denote $x_{1}^{i_{1}} x_{2}^{i_{2}} \ldots x_{g}^{i_{g}}$ and $S(X)=\left(s_{1}(X), \ldots, s_{g}(X)\right)$ where $s_{i}(X)$ is the $i$ th symmetric function on $g$ letters. Finally, let $e_{k}$ be the standard $k$ th basis vector in $\mathbb{R}^{g}$ and let $e$ be the $g$-tuple $(1,1, \ldots, 1)$.

Let $C$ be a complete nonsingular algebraic curve of genus $g$ defined over a field $K$, of characteristic zero. Let $A$ be the Jacobian of $C . A$ is an abelian variety of dimension $g$ defined over $K$. If $O$ is the local ring of functions defined at the origin, $\mathscr{M}$ its maximal ideal and $\widehat{O}$ the completion of $O$ in the $\mathscr{M}$-adic topology, then $\widehat{O}$ is isomorphic to $K[[X]]$ where $X=\left(x_{1}, \ldots, x_{g}\right)$ is a system of parameters at the origin. The group morphism $m: A \times A \rightarrow A$ induces a map $m^{*}: \widehat{O} \rightarrow \widehat{O} \times \widehat{O}$. Thus there are power series $\widehat{A_{i}}(X, Y) \in K[[X, Y]]$ such that $m_{i}^{*}=\widehat{A_{i}}(X, Y)$. Since $A$ is an abelian variety, it is easy to check that $\widehat{A}(X, Y)=\left(\widehat{A_{1}}(X, Y), \ldots, \widehat{A_{g}}(X, Y)\right)$ defines a commutative formal group, the formal group of the Jacobian of $C$. Changing the choice of parameters gives rise to an isomorphic though not strictly isomorphic formal group so we will make a definite choice of parameters.

Let $P_{0}$ be a $K$-rational point on $C$ which is not a Weierstrass point and let $t$ be a local parameter at $P_{0}$. Choose a basis $\left\{\eta_{1}, \ldots, \eta_{g}\right\}$ for the holomorphic differentials of $C$ such that the $K$-expansion of $\eta_{i}$ with respect to the parameter $t$ satisfies

$$
\eta_{i} \equiv(-t)^{i-1} d t \quad\left(\bmod t^{g} d t\right) .
$$

(See for example [1] to see that this can be done.) Let $l_{i}(t)=\int \eta_{i}$ be the integral of the formal power series $\eta_{i}$ satisfying $l_{i}(0)=0$ for $i=1, \ldots, g$ and let

$$
L_{i}\left(t_{1}, \ldots, t_{g}\right)=l_{i}\left(t_{1}\right)+\cdots+l_{i}\left(t_{g}\right) .
$$

$L_{i}$ is symmetric in $t_{1}, \ldots, t_{g}$ and thus $L_{i}\left(t_{1}, \ldots, t_{g}\right)=\mathscr{L}_{i}(S(T))$ where $\mathscr{L}_{i}$ is a power series is $g$ variables.

Let $\Lambda: C \rightarrow A$ be the canonical map defined over $K$ with $\Lambda\left(P_{0}\right)=$ origin of $A . \Lambda$ extends to a map $\Lambda^{(g)}: \operatorname{Sym}^{(g)} C \rightarrow A$ which is birational over $K$ and biregular in a neighborhood of $Q=\left(P_{0}, \ldots, P_{0}\right)$. This map induces isomorphisms between the completed local ring of $A$ and the completed local ring of $\operatorname{Sym}^{(g)} C$ and between the space of holomorphic differentials on $A$ and those on $\operatorname{Sym}^{(g)} C$. Thus we regard $\left(s_{1}(T), \ldots, s_{g}(T)\right)$ as a set of local parameters at the origin of $A$ and $d \mathscr{L}_{i}$ as the local expansion at the origin of a differential on $A$. Using this identification we have the following theorem: 
THEOREM 2. $\mathscr{L}(X)=\left(\mathscr{L}_{1}(X), \ldots, \mathscr{L}_{g}(X)\right)$ is the strict logarithm of the formal group of the Jacobian of $C$, i.e.,

$$
\widehat{A}(X, Y)=\mathscr{L}^{-1}(\mathscr{L}(X)+\mathscr{L}(Y))
$$

is the formal group of the Jacobian of $C$ and $\mathscr{L}(X) \equiv X(\bmod \operatorname{deg} 2)$.

Before proving the theorem, we need the following lemma expressing the sum of the $n$th powers of the variables $x_{i}$ as a polynomial in the symmetric functions.

LEMMA 1. Let $P_{n}(X)=x_{1}^{n}+\cdots+x_{g}^{n}$.

Then

$$
P_{n}(X)=n \sum_{\substack{I \\ N_{I}=n}} B(I)(S(X))^{I}
$$

Proof. $P_{n}(X)$ satisfies Newton's identities. That is,

$$
P_{n}(X)=\sum_{j=1}^{n-1}(-1)^{j+1} s_{j}(X) P_{n-j}(X)+(-1)^{n+1} n s_{n}(X)
$$

where $s_{j}(X)$ is defined to be 0 if $j>g$.

The lemma follows from these identities by induction. It is clearly true for $n=1$. Assume it is true for all integers less than $n$, then we have:

$$
\begin{aligned}
P_{n}(X)= & \sum_{j=1}^{n-1}(-1)^{j+1} s_{j}(X)\left((n-j) \sum_{\substack{I_{I^{\prime}}=n-j \\
I^{\prime}}} B\left(I^{\prime}\right)(S(X))^{I^{\prime}}\right) \\
& +(-1)^{n+1} n s_{n}(X) \\
= & \sum_{j=1}^{\min (n-1, g)}(-1)^{j+1}\left((n-j) \sum_{j=1}^{n-1} B\left(I^{\prime}\right)(S(X))^{I^{\prime}+e_{j}}\right) \\
& +(-1)^{n+1} n s_{n}(X) .
\end{aligned}
$$

Let $I=I^{\prime}+e_{j}$ then $B\left(I^{\prime}\right)=\left((-1)^{j+1} i_{j} /\left(i_{1}+\cdots+i_{g}-1\right)\right) B(I)$. 
As $j$ runs from 1 to $\min (n-1, g)$ and $I^{\prime}$ runs through all index sets with $N_{I}^{\prime}=n-j, I$ runs through index sets with $N_{I}=n$ except possibly $I=e_{n}$ where $n \leq g$.

If $n \geq g$ we get:

$$
\begin{aligned}
P_{n}(X) & =\sum_{\substack{I \neq e_{n} \\
N_{I}=n}} B(I)(S(X))^{I} \frac{1}{i_{1}+\cdots+i_{g}-1} \sum_{j=1}^{n-1}(n-j) i_{j}+B\left(e_{n}\right) n s_{n}(X) \\
& =\sum_{\substack{I \neq e_{n} \\
N_{I}=n}} B(I)(S(X))^{I} \frac{1}{i_{1}+\cdots+i_{g}-1} \sum_{j=1}^{g}(n-j) i_{j}+B\left(e_{n}\right) n s_{n}(X)
\end{aligned}
$$

since if $N_{I}=n \leq g, I \neq e_{n}, i_{j}=0$ for $j=n, \ldots, g$, we can replace $n-1$ by $g$ in the summation. Therefore

$$
\begin{aligned}
& =\sum_{\substack{I \neq e_{n} \\
N_{I}=n}} B(I)(S(X))^{I} \frac{1}{i_{1}+\cdots+i_{g}-1}\left(\sum_{j=1}^{g}\left(n i_{j}\right)-N_{I}\right)+B\left(e_{n}\right) n s_{n}(X) \\
& =\sum_{\substack{I \neq e_{n} \\
N_{I}=n}} n B(I)(S(X))^{I} \frac{1}{i_{1}+\cdots+i_{g}-1}\left(\sum_{j=1}^{g}\left(i_{j}\right)-1\right)+B\left(e_{n}\right) n s_{n}(X) \\
& =n \sum_{\substack{I \\
N_{I}=n}} B(I)(S(X))^{I} .
\end{aligned}
$$

If $n>g$, as $j$ runs from 1 to $n-1$ and $I^{\prime}$ runs through all index sets with $N_{I^{\prime}}=n-j, I$ runs through all index sets with $N_{I}=n$. Thus we have

$$
\begin{aligned}
P_{n}(X) & =\sum_{\substack{I \\
N_{l}=n}} B(I)(S(X))^{I} \frac{1}{i_{1}+\cdots+i_{g}-1}\left(\sum_{j=1}^{g}(n-j) i_{j}\right) \\
& =\sum_{\substack{I \\
N_{I}=n}} B(I)(S(X))^{I} \quad \text { as above. }
\end{aligned}
$$


Proof of Theorem 2. By the results of the last section, we must show that $\left\{d \mathscr{L}_{1}, \ldots, d \mathscr{L}_{g}\right\}$, where $d \mathscr{L}_{i}=\sum_{j=1}^{g}\left(\partial \mathscr{L}_{i}(X) / \partial x_{j}\right) d x_{j}$, is the standard basis for the invariant differentials on $\widehat{A}$. The canonical map $\Lambda$ from $C$ to $A$ induces a bijection $\Lambda_{*}: H^{0}\left(A, \Omega^{1}\right) \rightarrow H^{0}\left(C, \Omega^{1}\right)$ from the holomorphic differentials on $A$ to the holomorphic differentials on $C$. (See [9].) Since all holomorphic differentials on an abelian variety are invariant, it suffices to show that $\Lambda_{*} d \mathscr{L}_{i}$ is a holomorphic differential of $C$ for $i=1, \ldots, g$ and that $d \mathscr{L}_{i}(0)=d x_{i}$.

We write $\eta_{j}=\sum_{n=1}^{\infty} a_{j}(n) t^{n-1} d t$ where as above $t$ is a parameter at a rational non-Weierstrass point $P_{0}$ and $a_{j}(i)=(-1)^{j-1} \delta_{i j}$ for $i, j=1, \ldots, g$. Then

$$
\begin{aligned}
l_{j}(t) & =\sum_{n=1}^{\infty} a_{j}(n) / n t^{n}, \\
L_{j}(T) & =\sum_{n=1}^{\infty} \frac{a_{j}(n)}{n}\left(t_{1}^{n}+\cdots+t_{g}^{n}\right) .
\end{aligned}
$$

By Lemma 1,

$$
L_{j}(T)=\mathscr{L}_{j}(S(T))=\sum_{n=1}^{\infty} \sum_{\substack{I \\ N_{I}=n}} a_{j}(n) B(I)(S(T))^{I}
$$

i.e.,

$$
\begin{aligned}
\mathscr{L}_{j}(X) & =\sum_{I>0} a_{j}\left(N_{I}\right) B(I)(X)^{I}, \\
d \mathscr{L}_{j}(X) & =\sum_{k=1}^{g}\left(\sum_{I} i_{k} B(I) a_{j}\left(N_{I}\right)(X)^{I-e_{k}}\right) d x_{k}, \\
d \mathscr{L}_{j}(0) & =\sum_{k=1}^{g} B\left(e_{k}\right) a_{j}(k) d x_{k}=(-1)^{j+1}(-1)^{j-1} d x_{j}=d x_{j}
\end{aligned}
$$

There is a natural map $\varphi: C \rightarrow \operatorname{Sym}^{(g)} C$ which is given by the composition of the diagonal map $C \rightarrow C^{g}$ with the natural projection $C^{g} \rightarrow \operatorname{Sym}^{(g)} C$. The induced map on the completed local rings takes $x_{k}$ to $x_{k} \circ \Lambda=s_{k}(t, \ldots, t)=s_{k}(e) t^{k}$ and $(X \circ \Lambda)^{I}=(S(e))^{I} t^{N_{I}}$. 


$$
\begin{aligned}
\Lambda_{*} d \mathscr{L}_{j}(X) & =\sum_{k=1}^{g} \sum_{I} i_{k} B(I) a_{j}\left(N_{I}\right)(X \circ \Lambda)^{I-e_{k}} d\left(x_{k} \circ \Lambda\right) \\
& =\sum_{k=1}^{g} \sum_{I} i_{k} B(I) a_{j}\left(N_{I}\right)(S(e))^{I-e_{k}}\left(t^{N_{I-e_{k}}}\right)\left(k s_{k}(e) t^{k-1}\right) d t \\
& =\sum_{I} B(I)\left(\sum_{k=1}^{g} k i_{k}\right) a_{j}\left(N_{I}\right)(S(e))^{I}\left(t^{N_{I}-1}\right) d t \\
& =\sum_{I} B(I) N_{I} a_{j}\left(N_{I}\right)(S(e))^{I}\left(t^{N_{I}-1}\right) d t \\
& =\sum_{n=1}^{\infty}\left(\begin{array}{c}
\sum_{I} B(I) n(S(e))^{I} \\
N_{I}=n
\end{array}\right) a_{j}(n) t^{n-1} d t \\
& =\sum_{n=1}^{\infty} P_{n}(e) a_{j}(n) t^{n-1} d t=g \eta_{j} .
\end{aligned}
$$

Note: if a different basis of differentials is used we still have $\mathscr{L}(X)$ is a logarithm of the formal group of the Jacobian but it is not the strict logarithm.

III. The formal group of the Jacobian of $X_{0}(l)$. Let $l$ be an odd positive prime and

$$
\Gamma_{0}(l)=\left\{\left(\begin{array}{ll}
a & b \\
c & d
\end{array}\right) \in \mathrm{SL}_{2}(\mathbb{Z}) \mid c \equiv 0(\bmod l)\right\}
$$

Let $\mathscr{H}$ be the complex upper half-plane. Let $Y_{0}(l)=\mathscr{H} / \Gamma_{0}(l)$. Then $Y_{0}(l)$ can be compactified by the addition of finitely many cusps. The resulting complex curve has a complete non-singular model defined over $\mathbb{Q}$ which we denote by $X_{0}(l)$ (Shimura [12]). The genus of $X_{0}(l)$ is

$$
\begin{cases}{\left[\frac{l+1}{12}\right]} & \text { if } 12 \nmid l-1 \\ {\left[\frac{l+1}{12}\right]-1} & \text { if } 12 \mid l-1 .\end{cases}
$$

Atkin and Ogg have shown that the cusp at $\infty$ is not a Weierstrass point on $X_{0}(l)$, (Ogg [10]), so we will use this as base point to construct the formal group of the Jacobian of $X_{0}(l)$. 
If $f(z)=\sum_{n=1}^{\infty} a_{n} e^{2 \pi i z n}$ is the Fourier expansion at $i \infty$ of a cusp form of weight 2 for $\Gamma_{0}(l)$ then a parameter $q$ can be chosen on $X_{0}(l)$ at the cusp at $i \infty$ so that

$$
\omega=2 \pi i f(z) d z=\sum_{n=1}^{\infty} a_{n} q^{n-1} d q
$$

is the expansion at $i \infty$ of a holomorphic differential on $X_{0}(l)$.

Let $\left\{f_{1}, \ldots, f_{g}\right\}$ be a $\mathbb{Q}$-basis for the cusp forms of weight 2 on $\Gamma_{0}(l)$ with Fourier expansions $\sum c_{i}(n) e^{2 \pi i z n}$ at $i \infty$ and $\left[c_{i}(j)\right]=\operatorname{Id}_{g}$, the $g \times g$ identity matrix. Let $\left\{\omega_{1}, \ldots, \omega_{g}\right\}$ be the corresponding basis for the holomorphic differentials on $X_{0}(l)$. Let

$$
\mathscr{L}(X)=\left(\mathscr{L}_{1}(X), \ldots, \mathscr{L}_{g}(X)\right)
$$

be the logarithm map as given in the construction above. In this case the Jacobian matrix of $\mathscr{L}, J(\mathscr{L})=\left[(-1)^{i+1} \delta_{i j}\right]$.

Since the cusp forms of weight 2 on $\Gamma_{0}(l)$ have a $\mathbb{Z}$ basis also, (Shimura [12]), there are only finitely many primes which divide the denominators of $\left\{c_{i}(n)\right\}$ for $i=1, \ldots, g$ and $n \in \mathbb{Z}^{+}$. Let $S$ be this finite set of primes.

THEOREM 3. Let $l \in \mathbb{Z}$ be a prime. If the formal group of the Jacobian of the modular curve $X_{0}(l)$ is given by $\mathfrak{J}(X, Y)=$ $\mathscr{L}^{-1}(\mathscr{L}(X)+\mathscr{L}(Y))=\left(J_{1}(X, Y), \ldots, J_{g}(X, Y)\right)$, then $J_{i}(X, Y) \in$ $\mathbb{Z}_{p}[[X, Y]]$ for all primes $p \notin S$.

Before proving this we will need the following lemmas. Let $M_{p}=$ $\left[c_{i}(j p)+p c_{i}(j / p)\right]$ for $p \neq l$, and $M_{l}=\left[c_{i}(j l)\right]$ where $c_{i}(j / p)=0$ if $p \nmid j$.

LEMMA 2.

$$
\left[\begin{array}{c}
c_{1}(n p) \\
\vdots \\
c_{g}(n p)
\end{array}\right]-M_{p}\left[\begin{array}{c}
c_{1}(n) \\
\vdots \\
c_{g}(n)
\end{array}\right]+p\left[\begin{array}{c}
c_{1}(n / p) \\
\vdots \\
c_{g}(n / p)
\end{array}\right]=0
$$

for all $n \in \mathbb{Z}^{+}$and for all primes $p$.

Proof. Let $T_{p}$ and $U_{p}$ be the standard Hecke and Atkin operators. Since $f_{i}$ is a cusp form of weight two on $\Gamma_{0}(l), T_{p}\left(f_{i}\right)$ for all primes $p \neq l$ and $U_{l}\left(f_{i}\right)$ are also cusp forms of weight two on $\Gamma_{0}(l)$ (Atkin 
[2]). Since $\left\{f_{1}, \ldots, f_{g}\right\}$ is a basis we have

$$
\begin{aligned}
& {\left[\begin{array}{c}
T_{p}\left(f_{1}\right) \\
\vdots \\
T_{p}\left(f_{g}\right)
\end{array}\right]=C_{p}\left[\begin{array}{c}
f_{1} \\
\vdots \\
f_{g}
\end{array}\right] \quad \text { for some } C_{p} \in M_{n}(\mathbb{Q}) \quad \text { and }} \\
& {\left[\begin{array}{c}
U_{l}\left(f_{1}\right) \\
\vdots \\
U_{l}\left(f_{g}\right)
\end{array}\right]=C_{l}\left[\begin{array}{c}
f_{1} \\
\vdots \\
f_{g}
\end{array}\right] \quad \text { for some } C_{l} \in M_{n}(\mathbb{Q}) .}
\end{aligned}
$$

Thus the Fourier coefficients of the $f_{i}$ satisfy the following:

$$
\left[\begin{array}{c}
c_{1}(n p)+p c_{1}(n / p) \\
\vdots \\
c_{g}(n / p)+p c_{g}(n / p)
\end{array}\right]=C_{p}\left[\begin{array}{c}
c_{1}(n) \\
\vdots \\
c_{g}(n)
\end{array}\right] \quad \begin{aligned}
& \text { for all } p \neq l \text { and } \\
& \text { for all } n \in \mathbb{Z}^{+}
\end{aligned}
$$

and

$$
\left[\begin{array}{c}
c_{1}(n l) \\
\vdots \\
c_{g}(n l)
\end{array}\right]=C_{l}\left[\begin{array}{c}
c_{1}(n) \\
\vdots \\
c_{g}(n)
\end{array}\right] \quad \text { for all } n \in \mathbb{Z}^{+}
$$

Evaluating at $n=1, \ldots, g$ gives $C_{p}=M_{p}$ and $C_{l}=M_{l}$.

The following is a special case of a theorem of T. Honda. (Honda [6].) Hazewinkel ([5] page 59) gives a similar result in a functional equation lemma. Let $U_{n}=\left\{n \times n\right.$ matrices over $\left.\mathbb{Z}_{p}[[t]]\right\}$. We say $u \in U_{n}$ is a special element if $u \equiv p I_{n}(\bmod$ degree 1$)$. For $f(X)=$ $\left(f_{1}(X), \ldots, f_{n}(X)\right), f_{i}(X) \in \mathbb{Q}_{p}[[X]], f_{i}(0)=0$ and $u=\sum C_{j} t^{j} \in$ $U_{n}$, we define $(u * f)(X)=\sum C_{j} f\left(X^{p^{j}}\right)$.

Lemma 3 (Honda [6]). Let $f(X)=\left(f_{1}(X), \ldots, f_{n}(X)\right), f_{i}(X) \in$ $\mathbb{Q}_{p}[[x]]$ with $f_{i}(0)=0$. Assume $J(f)$ is an invertible matrix in $M_{n}\left(\mathbb{Z}_{p}\right)$. If there exists a special element $u \in U_{n}$ such that $u * f \equiv 0$ $(\bmod p)$ then $F(X, Y)=f^{-1}(f(X)+f(Y))$ is a commutative formal group of dimension $n$ defined over $\mathbb{Z}_{p}$. Moreover, if $g(X)=$ $\left(g_{1}(X), \ldots, g_{n}(X)\right)$, with $g_{i}(X) \in \mathbb{Q}_{p}[[X]]$ and $g_{i}(0)=0$ also has $J(g)$ invertible in $M_{n}\left(\mathbb{Z}_{p}\right)$ and $u * g \equiv 0(\bmod p)$ for the same element $u$, then $G(X, Y)=g^{-1}(g(X)+g(Y))$ is isomorphic to $F(X, Y)$ over $\mathbb{Z}_{p}$.

LEMMA 4. $p B\left(I / p^{r}\right)-B\left(I / p^{r+1}\right) \equiv 0\left(\bmod p \mathbb{Z}_{p}\right)$ for any $r$ and for any index set $I=\left(i_{1}, \ldots, i_{g}\right)$ where $B\left(I / p^{r}\right)=0$ if $p^{r} \nmid I$ (i.e., if $p^{r} \nmid i_{k}$ for some $\left.k\right)$. 
Proof. Let $n \in \mathbb{Z}$ with $p \nmid n$. Then by induction we see

$$
\left(x_{1}+\cdots+x_{g}\right)^{p^{a} n} \equiv\left(x_{1}^{p}+\cdots+x_{g}^{p}\right)^{p^{a-1} n} \quad\left(\bmod p^{a}\right) .
$$

Therefore by the multinomial theorem,

$$
\sum_{\substack{R \\ n_{g}=p^{a} n}} \frac{\left(p^{a} n\right) !}{R !} X^{R} \equiv \sum_{\substack{T \\ t_{1}+\cdots+t_{g}=p^{a-1} n}} \frac{\left(p^{a-1} n\right) !}{T !} X^{p T}\left(\bmod p^{a}\right) .
$$

Let $J=\left(j_{1}, \ldots, j_{g}\right)$ be an index set such that $p \nmid j_{k}$ for some $k$ and $p^{b}\left(j_{1}+\cdots+j_{g}\right)=p^{a} n$ for some $b, 1 \leq b \leq a$. By comparing the coefficients of $X^{p^{b} J}$ above we have:

$$
\begin{gathered}
\frac{\left(p^{a} n\right) !}{\left(p^{b} J\right) !} \equiv \frac{\left(p^{a-1} n\right) !}{\left(p^{b-1} J\right) !}\left(\bmod p^{a}\right) \\
\frac{p^{a} n}{p^{b} j_{k}}\left(\frac{\left(p^{a} n-1\right) !}{\left(p^{b} j_{1} ! \cdots\left(p^{b} j_{k}-1\right) ! \cdots\left(p^{b-1} j_{g}\right) !\right.}\right) \\
\equiv \frac{p^{a-1} n}{p^{b-1} j_{k}}\left(\frac{\left(p^{a-1} n-1\right) !}{\left(p^{b-1} j_{1}\right) ! \cdots\left(p^{b-1} j_{k}-1\right) ! \cdots\left(p^{b-1} j_{g}\right) !}\right)\left(\bmod p^{a}\right)
\end{gathered}
$$

Since $p \nmid n$ and $p \nmid j_{k}$ we have:

$$
\begin{aligned}
& \frac{\left(p^{a} n-1\right) !}{\left(p^{b} j_{1}\right) ! \cdots\left(p^{b} j_{k}-1\right) ! \cdots\left(p^{b} j_{g}\right) !} \\
& \equiv \frac{\left(p^{a-1} n-1\right) !}{\left(p^{b-1} j_{1}\right) ! \cdots\left(p^{b-1} j_{k}-1\right) ! \cdots\left(p^{b-1} j_{g}\right) !}\left(\bmod p^{b} \mathbb{Z}_{p}\right) .
\end{aligned}
$$

Now let $I=\left(i_{1}, \ldots, i_{g}\right)$ be any index set and fix $r \in \mathbb{Z}$. If $p^{r} \nmid I$ then $p B\left(I / p^{r}\right)-B\left(I / p^{r+1}\right)=0$. Thus assume $I=p^{b} J$ where $p \nmid J$ and $b \geq r$.

If $b=r$,

$$
p B\left(I / p^{r}\right)-B\left(I / p^{r+1}\right)=p B\left(I / p^{r}\right)=p B(J)= \pm\left(p / j_{k}\right) j_{k} B(J)
$$

where $p \nmid j_{k}$ and $j_{k} B(J)$ is a multinomial coefficient. Thus $p B\left(I / p^{r}\right)$ $-B\left(I / p^{r+1}\right) \equiv 0\left(\bmod p \mathbb{Z}_{p}\right)$.

Assume $b>r$.

$$
\begin{aligned}
& p B\left(I / p^{r}\right)-B\left(I / p^{r+1}\right)=p B\left(p^{b-r} J\right)-B\left(p^{b-r-1} J\right) \\
& =\frac{ \pm 1}{p^{b-r-1} j_{k}}\left(\frac{\left(p^{a} n-1\right) !}{\left(p^{b-r} j_{1}\right) ! \cdots\left(p^{b-r} j_{k}-1\right) ! \cdots\left(p^{b-r} j_{g}\right) !}\right. \\
& \left.\quad-\frac{\left(p^{a-1} n-1\right) !}{\left(p^{b-r-1} j_{1}\right) ! \cdots\left(p^{b-r-1} j_{k}-1\right) ! \cdots\left(p^{b-r-1} j_{g}\right) !}\right)
\end{aligned}
$$

where $p^{a} n=p^{b-r}\left(j_{1}+\cdots+j_{g}\right)$ and $p \nmid n$.

Hence $p B\left(I / p^{r}\right)-B\left(I / p^{r+1}\right) \equiv 0\left(\bmod p \mathbb{Z}_{p}\right)$ by $(4)$ above. 
THEOREM 4. For any prime $p \notin S$, let $u_{p}=p I_{g}-M_{p} t+t^{2}$. Then $u_{p}$ is a special element in the sense of Honda and $u_{p} * \mathscr{L} \equiv 0(\bmod p)$.

Theorem 3 follows immediately from this result and Lemma 3.

Proof. Recall that $\mathscr{L}_{j}(X)=\sum_{I>0} B(I) c_{j}\left(N_{I}\right) X^{I}$. Thus

$$
\begin{aligned}
u_{p} * \mathscr{L}= & p\left(\begin{array}{c}
\mathscr{L}_{1}(X) \\
\vdots \\
\mathscr{L}_{g}(X)
\end{array}\right)-M_{p}\left(\begin{array}{c}
\mathscr{L}_{1}\left(X^{p}\right) \\
\vdots \\
\mathscr{L}_{g}\left(X^{p}\right)
\end{array}\right)+\left(\begin{array}{c}
\mathscr{L}_{1}\left(X^{p^{2}}\right) \\
\vdots \\
\mathscr{L}_{g}\left(X^{p^{2}}\right)
\end{array}\right) \\
= & p\left(\begin{array}{c}
\sum_{I>0} B(I) c_{1}\left(N_{I}\right) X^{I} \\
\vdots \\
\sum_{I>0} B(I) c_{g}\left(N_{I}\right) X^{I}
\end{array}\right)-M_{p}\left(\begin{array}{c}
\sum_{I>0} B(I) c_{1}\left(N_{I}\right) X^{p I} \\
\vdots \\
\sum_{I>0} B(I) c_{g}\left(N_{I}\right) X^{p I}
\end{array}\right) \\
& +\left(\begin{array}{l}
\sum_{I>0} B(I) c_{1}\left(N_{I}\right) X^{p^{2} I} \\
\vdots \\
\sum_{I>0} B(I) c_{g}\left(N_{I}\right) X^{p^{2} I}
\end{array}\right) .
\end{aligned}
$$

Using Lemma 2,

$$
\begin{aligned}
M_{p}\left(\begin{array}{c}
\sum_{I>0} B(I) c_{1}\left(N_{I}\right) X^{p I} \\
\vdots \\
\sum_{I>0} B(I) c_{g}\left(N_{I}\right) X^{p I}
\end{array}\right)= & \left(\begin{array}{c}
\sum_{I>0} B(I) c_{1}\left(p N_{I}\right) X^{p I} \\
\vdots \\
\sum_{I>0} B(I) c_{g}\left(p N_{I}\right) X^{p I}
\end{array}\right) \\
& +p\left(\begin{array}{c}
\sum_{I>0} B(I) c_{1}\left(\frac{1}{p} N_{I}\right) X^{p I} \\
\vdots \\
\sum_{I>0} B(I) c_{g}\left(\frac{1}{p} N_{I}\right) X^{p I}
\end{array}\right) .
\end{aligned}
$$

Let $J=p I$. Then $N_{J}=p N_{I}$. 
Thus we have:

$$
\begin{aligned}
u_{p} * \mathscr{L}=p & \left(\begin{array}{c}
\sum_{I>0} B(I) c_{1}\left(N_{I}\right) X^{I} \\
\vdots \\
\sum_{I>0} B(I) c_{g}\left(N_{I}\right) X^{I}
\end{array}\right)-\left(\begin{array}{c}
\sum_{I>0} B(I) c_{1}\left(p N_{I}\right) X^{p I} \\
\vdots \\
\sum_{I>0} B(I) c_{g}\left(p N_{I}\right) X^{p I}
\end{array}\right) \\
& -p\left(\begin{array}{c}
\sum_{\substack{J=p I \\
I>0}} B\left(\frac{J}{p}\right) c_{1}\left(\frac{1}{p^{2}} N_{j}\right) X^{J} \\
\sum_{\substack{J=p I \\
I>0}} B\left(\frac{J}{p}\right) c_{g}\left(\frac{1}{p^{2}} N_{J}\right) X^{J}
\end{array}\right) \\
& +\left(\begin{array}{c}
\left.\sum_{\substack{J=p I \\
I>0}} B\left(\frac{J}{p}\right) c_{1}\left(\frac{I}{p} N_{J}\right) X^{p J}\right) \\
\left.\sum_{\substack{J=p I \\
I>0}} B\left(\frac{J}{p}\right) c_{1}\left(\frac{I}{p} N_{J}\right) X^{p J}\right)
\end{array}\right.
\end{aligned}
$$

where $B\left(J / p^{i}\right)=0$ if $p^{i} \nmid J$.

$=\left(\begin{array}{c}\sum_{I>0}\left(p B(I)-B\left(\frac{I}{p}\right)\right) c_{g}\left(N_{I}\right) X^{I} \\ \sum_{I>0}\left(p B(I)-B\left(\frac{I}{p}\right)\right) c_{g}\left(N_{I}\right) X^{I}\end{array}\right)$

$$
-\left(\begin{array}{c}
\sum_{\substack{J=p I \\
I>0}}\left(p B\left(\frac{J}{p}\right)-B\left(\frac{J}{p^{2}}\right)\right) c_{1}\left(\frac{1}{p^{2}} N_{J}\right) X^{J} \\
\sum_{\substack{J=p I \\
I>0}}\left(p B\left(\frac{J}{p}\right)-B\left(\frac{J}{p^{2}}\right)\right) c_{g}\left(\frac{1}{p^{2}} N_{J}\right) X^{J}
\end{array}\right)
$$

by Lemma 4 .

REMARKs. (1) If $p>g, M_{p}$ reduces to the matrix $\left[c_{i}(j p)\right]$, the Hasse-Witt matrix of the curve. The special element $u_{p}$ is then a generalization of the special element found by Honda ([7]) in the elliptic 
curve case, namely $u_{p}=p-a_{p} t+t^{2}$ where $a_{p}$ is the Hasse invariant of the elliptic curve.

(2) The cusp forms of weight 2 on $\Gamma_{0}(l)$ also have a $\mathbb{Z}$-basis. (See Shimura [12]). If $\left\{f_{1}, \ldots, f_{g}\right\}$ is this basis with $f_{i}(q)=\sum a_{i}(n) q^{n}$, then the primes in the set $S$ are precisely those primes which divide the determinant of the matrix $\left[a_{i}(j)\right]$.

3. The author wishes to thank M. Rosen and J. Lubin for many helpful discussions about this material. In particular, it was J. Lubin who suggested the construction given in $§ I I$.

\section{REFERENCES}

[1] E. Arbarello, M. Cornalba, P. A. Griffiths and J. Harris, Geometry of Algebraic Curves, Volume I, Springer-Verlag, 1985.

[2] A. Atkin and J. Lehner, Hecke operators on $\Gamma_{0}(m)$, Math. Ann., 185 (1970), 134-160.

[3] E. V. Flynn, The Jacobian and formal group of a curve of genus 2 over an arbitrary ground field, Math. Proc. Camb. Phil. Soc., 107 (1990), 425-441.

[4] D. Grant, Formal groups in genus 2, J. Reine Angew. Math., (1990), 96-121.

[5] M. Hazewinkel, Formal Groups and Applications, Academic Press, 1978.

[6] T. Honda, On the theory of commutative formal groups, J. Math. Soc. Japan, 22 (1970), 213-246.

[7] _ Formal groups and zeta functions, Osaka J. Math., 5 (1968), 199-213.

[8] J. S. Milne, Abelian Varieties, In Arithmetic Geometry, G. Cornell and J. Silverman Eds., Springer-Verlag 1986.

[9] _ Jacobian Varieties, In Arithmetic Geometry, G. Cornell and J. Silverman Eds., Springer-Verlag 1986.

[10] A. Ogg, On the Weierstrass points of $X_{0}(N)$, Illinois J. Math., 22 (1978), 31-35.

[11] S. Shatz, Group Schemes, Formal Groups and p-Divisible Groups, in Arithmetic Geometry, G. Cornell and J. Silverman Eds., Springer-Verlag 1986.

[12] G. Shimura, Introduction to the Arithmetic Theory of Automorphic Functions, Princeton University Press, 1971.

[13] J. Silverman, The Arithmetic of Elliptic Curves, Springer-Verlag, 1986.

Received August 4, 1990.

College of the Holy Cross

WORCESTER, MA 01610 



\title{
PACIFIC JOURNAL OF MATHEMATICS
}

\author{
Founded by
}

E. F. BECKENBACH (1906-1982) F. WoLF (1904-1989)

\section{EDITORS}

$\begin{array}{ll}\begin{array}{l}\text { V. S. VARADARAJAN } \\ \text { (Managing Editor) }\end{array} & \begin{array}{l}\text { NiCHOLAS ERCOLANI } \\ \text { University of Arizona }\end{array} \\ \text { University of California } & \text { Tucson, AZ 85721 } \\ \text { Los Angeles, CA 90024-1555 } & \text { ercolani@math.arizona.edu } \\ \text { vsv@math.ucla.edu } & \text { R. FinN } \\ \text { HERBERT CLEMENS } & \text { Stanford University } \\ \text { University of Utah } & \text { Stanford, CA 94305 } \\ \text { Salt Lake City, UT 84112 } & \text { finn@gauss.stanford.edu } \\ \text { clemens@math.utah.edu } & \text { VAUGHAN F. R. JoNEs } \\ \text { F. MichAEL CHRIsT } & \text { University of California } \\ \text { University of California } & \text { Berkeley, CA 94720 } \\ \text { Los Angeles, CA 90024-1555 } & \text { vfr@math.berkeley.edu } \\ \text { christ@math.ucla.edu } & \text { STEVEN KERCKHoFF } \\ \text { THomas ENRIGHT } & \text { Stanford University } \\ \text { University of California, San Diego } & \text { Stanford, CA 94305 } \\ \text { La Jolla, CA 92093 } & \text { spk@gauss.stanford.edu } \\ \text { tenright@ucsd.edu } & \end{array}$

\author{
C. C. MOORE \\ University of California \\ Berkeley, CA 94720
}

MaRTin ScharlemanN

University of California

Santa Barbara, CA 93106

mgscharl@henri.ucsb.edu

\author{
HAROLD STARK \\ University of California, San Diego \\ La Jolla, CA 92093
}

\begin{tabular}{ll}
\multicolumn{1}{c}{ SUPPORTING } & INSTITUTIONS \\
UNIVERSITY OF ARIZONA & UNIVERSITY OF OREGON \\
UNIVERSITY OF BRITISH COLUMBIA & UNIVERSITY OF SOUTHERN CALIFORNIA \\
CALIFORNIA INSTITUTE OF TECHNOLOGY & STANFORD UNIVERSITY \\
UNIVERSITY OF CALIFORNIA & UNIVERSITY OF HAWAII \\
MONTANA STATE UNIVERSITY & UNIVERSITY OF TOKYO \\
UNIVERSITY OF NEVADA, RENO & UNIVERSITY OF UTAH \\
NEW MEXICO STATE UNIVERSITY & WASHINGTON STATE UNIVERSITY \\
OREGON STATE UNIVERSITY & UNIVERSITY OF WASHINGTON
\end{tabular}

The Supporting Institutions listed above contribute to the cost of publication of this Journal, but they are not owners or publishers and have no responsibility for its content or policies.

Mathematical papers intended for publication in the Pacific Journal of Mathematics should be in typed form or offset-reproduced (not dittoed), double spaced with large margins. Please do not use built up fractions in the text of the manuscript. However, you may use them in the displayed equations. Underline Greek letters in red, German in green, and script in blue. The first paragraph must be capable of being used separately as a synopsis of the entire paper. In particular it should contain no bibliographic references. Please propose a heading for the odd numbered pages of less than 35 characters. Manuscripts, in triplicate, may be sent to any one of the editors. Please classify according to the 1991 Mathematics Subject Classification scheme which can be found in the December index volumes of Mathematical Reviews. Supply name and address of author to whom proofs should be sent. All other communications should be addressed to the managing editor, or Julie Speckart, University of California, Los Angeles, California 90024-1555.

There are page-charges associated with articles appearing in the Pacific Journal of Mathematics. These charges are expected to be paid by the author's University, Government Agency or Company. If the author or authors do not have access to such Institutional support these charges are waived. Single authors will receive 50 free reprints; joint authors will receive a total of 100 free reprints. Additional copies may be obtained at cost in multiples of 50 .

The Pacific Journal of Mathematics (ISSN 0030-8730) is published monthly except for July and August. Regular subscription rate: $\$ 190.00$ a year (10 issues). Special rate: $\$ 95.00$ a year to individual members of supporting institutions.

Subscriptions, orders for numbers issued in the last three calendar years, and changes of address should be sent to Pacific Journal of Mathematics, P.O. Box 969, Carmel Valley, CA 93924, U.S.A. Old back numbers obtainable from Kraus Periodicals Co., Route 100, Millwood, NY 10546.

The Pacific Journal of Mathematics at P.O. Box 969, Carmel Valley, CA 93924 (ISSN 0030-8730) is published monthly except for July and August. Second-class postage paid at Carmel Valley, California 93924, and additional mailing offices. Postmaster: send address changes to Pacific Journal of Mathematics, P.O. Box 969, Carmel Valley, CA 93924.

PUBLISHED BY PACIFIC JOURNAL OF MATHEMATICS, A NON-PROFIT CORPORATION Copyright $(\mathcal{1} 1993$ by Pacific Journal of Mathematics 


\section{PACIFIC JOURNAL OF MATHEMATICS}

Volume $157 \quad$ No. $2 \quad$ February 1993

Strong integral summability and the Stone-Čech compactification of the half-line

JEFF CONNOR and MARY ANNE SWARDSON

The endlich Baer splitting property

225

THEODORE GERARD FATICONI

The formal group of the Jacobian of an algebraic curve

MARGARET N. FREIJE

Concordances of metrics of positive scalar curvature

PAWEL GAJER

Explicit construction of certain split extensions of number fields and constructing cyclic classfields

STANLEY JOSEPH GURAK

Asymptotically free families of random unitaries in symmetric groups

ALEXANDRU MiHAi NiCA

On purifiable subgroups and the intersection problem

TAKASHI OKUYAMA

On the incidence cycles of a curve: some geometric interpretations

LUCIANA RAMELLA

On some explicit formulas in the theory of Weil representation

R. RANGA RAO

An analytic family of uniformly bounded representations of a free product of 373 discrete groups

JANUSZ WYSOCZAŃSKI

Errata: "Dentability, trees, and Dunford-Pettis operators on $L_{1}$ "

MARIA GIRARDI and ZHIBAO HU

Errata: "Poincaré cobordism exact sequences and characterisation" 